\title{
Infrared temperature measurement of vaporizing droplets
}

\author{
by L. NANA $\left(^{\star}\right)$, J. FARRE $\left(^{*}\right)$ and A. GIOVANNINI $\left({ }^{\star \star}\right)$
}

Centre d'Etudes et de Recherches de Toulouse (CERT-ONERA), DERMES, 2 avenue Edouard Belin, BP 4025, 31055 Toulouse Cedex, France. And:

(*) Ecole Nationale Supérieure de l'Aéronautique et de l'Espace, Département Electronique, 10 avenue Edouard Belin, BP 4032, 31055 Toulouse Cedex, France.

(**) Université Paul Sabatier, UFR MIG, Département de Mécanique, 118 Route de Narbonne, 31062 Toulouse Cedex, France.

\section{Abstract}

Our objective, is the measurement using infrared thermography (IR), of the temperature field of a methanol droplet. A mathematical model has been devised to give the performance (signal-noise ratio) of a proposed experimental system that measures the temperature of a droplet using IR thermography. Based on that simulation, an experimental system working in the long wavelength range (8-12 $\mu \mathrm{m})$ has been built and characterized in terms of temperature calibration and in terms of space and temperature resolutions. In order to validate the in measurement, the temperature evolution of a droplet of one millimeter in diameter has been measured with the IR system and with a thermocouple. For that purpose, the droplet fixed at the thermocouple is heated by a laser beam and cooled in still ambient air. The results obtained from both experimental techniques have shown very good agreement, along with the results obtained from a numerical model of the problem.

\section{Nomenclature}

$\begin{array}{ll}A_{d} & \text { detector area } \\ \Delta f & \text { noise equivalent bandwidth } \\ X_{o b j} & \text { object-entrance pupil distance } \\ D p & \text { entrance pupil diameter } \\ D_{o b j} & \text { object diameter in the detector field of view } \\ T & \text { object temperature }[\mathrm{K}] \\ T_{b} & \text { background temperature }[\mathrm{K}] \text { of the detector (user conditions) } \\ T_{b m} & \text { background temperature of the detector (manufacturer conditions) } \\ \lambda & \text { wavelength } \\ \theta_{d} & \text { semi-angle of view of the cooled detector } \\ \theta_{d m} & \text { semi-angle of view of the cooled detector in the manufacturer measurement conditions } \\ \lambda_{1}-\lambda 2 & \text { spectral bandwidth } \\ T_{a}\left(\lambda, X_{o b j-d \theta t}\right) & \text { spectral atmospheric transmission } \\ T_{o}(\lambda) & \text { spectral optical transmission } \\ E(\lambda) & \text { object spectral emissivity } \\ L_{B B}(\lambda, T) & \text { blackbody spectral luminance at temperature } T \\ R(\lambda) & \text { detector spectral responsivity } \\ D^{*}\left(\lambda, T_{b m}, \theta_{d m}\right) & \text { detector spectral detectivity in the manufacturer measurement conditions } \\ D^{*}\left(\lambda, T_{b}, \theta_{d}\right) & \text { detector spectral detectivity in the user measurement conditions } \\ F^{*} & \text { system figure of merit }\end{array}$

\section{Introduction}

Inside aeronautical and automotive engines, fuel is often injected in the combustion chambers in a dispersed liquid phase. Individual droplets have different velocity and temperature than the gas particles. In order to predict efficiency of the combustion and the formation of the pollutant, complete modelling of a single droplet behaviour, and consequently of the reactor, is necessary. This model has to take into account the temperature evolution during transient heating, vaporization and combustion phases. 


\section{http://dx.doi.org/10.21611/qirt.1992.015}

An isolated droplet is very often studied by photographic observation, a technique that yields for both the diameter and trajectory. However the temperature, a critical information for the validation of heat and mass transfer models, is not very often measured. Our first objective is to realize an infrared thermographic system in order to quantify the temperature field of a methanol droplet, which is the fluid of substitution of the fuel, with a thermal resolution of the system lower than one degree centigrade.

In order to validate the IR measurement, a droplet of about one millimeter in diameter is fixed on a thermocouple in still ambient air. By heating the droplet with a laser beam and letting it cool in the ambient air, comparisons on IR measurements can thus be made with the thermocouple measurements. The measurements are also compared with the results obtained from a numerical model of the problem.

\section{Definition of the measurement system}

The system is built from an infrared detector cooled by liquid nitrogen, an optical lens and a set of scanning mirrors $X, Y$ allowing the definition of the field of view. The measurement sensitivity is intrinsically linked to the spectral emissivity of the object viewed. Assuming that the droplet is spherical, the knowledge of the spectral variation of the complex refractive index of the methanol allows to compute the absorbed, and in consequence the emitted, fraction of the energy incident on the droplet as a plane wave [1]. As shown on figure 4, one can observe two bands B1 $(2-4 \mu \mathrm{m})$ and B2 $(6-11 \mu \mathrm{m})$ where emissivity is close to unity.

To analyse the influence of every parameter of the system on the overall system performance, we developed a specific software that analyse the variations of NETD (Noise Equivalent Temperature Difference) relative to the droplet at a temperature $T$ on a thermal background at $T b$. Referring to the parameters given in nomenclature, the formulation of the NETD is:

$$
N E T D[K]=\frac{16\left(A_{d} \Delta f\right)^{1 / 2} \cdot X_{o b j}^{2} \cdot\left(1+\frac{D_{p}^{2}}{2 X_{o b j}^{2}}\right)}{\left(\pi D_{p} D_{o b j}\right)^{2}} \times \frac{1}{F^{*}}
$$

with

$$
F^{*}=\int_{\lambda_{1}}^{\lambda_{2}} \frac{\partial}{\partial T}\left[L_{B B}(\lambda, T)\right] \cdot E(\lambda) \cdot T_{a}\left(\lambda, X_{\text {obj-det }}\right) \cdot T_{0}\left(D^{*}\left(\lambda, T_{b}, \theta_{d}\right) d \lambda\right.
$$

and

$$
D^{*}\left(\lambda, T_{b}, \theta_{d}\right)=D^{*}\left(\lambda, T_{b m}, \theta_{d m}\right) \frac{\sin \left(\theta_{d m}\right)}{\sin \left(\theta_{d}\right)}\left|\frac{\left.\int_{\lambda_{1}}^{\lambda_{2}} R(\lambda) \cdot L_{b b}\left(\lambda, T_{b m}\right) d \lambda\right|^{1 / 2}}{\int_{\lambda_{1}}^{\lambda_{2}} R(\lambda) \cdot L_{b b}\left(\lambda, T_{b}\right) d \lambda}\right|^{\int_{1}}
$$

When this software is runned for the normal droplet conditions and for the two bands B1 and $\mathrm{B} 2$, the band $\mathrm{B} 2$ gives a lower NETD than $\mathrm{B} 1$ by approximately a factor of 3 . The system was thus designed in the long wave band, using the following components : a ZnSe lens ( $f \#=$ 2.88), a HgCdTe detector $(8-10,5 \mu \mathrm{m}, 77 \mathrm{~K})$ completed by amplification and filtering $(\Delta f=1.5 \mathrm{KHz})$ electronics and a couple of mobile $X, Y$ mirrors. The acquisition and digitization (12 bits) of the signal and the mirror commands, are performed by a Tandon $P C 386-20 \mathrm{MHz}$. 
Figure 1 shows the measurement principle of the experimental IR system. The droplet is placed before an extended blackbody and the measurement is performed on differential mode : the detector viewing on a scanned line successively the blackbody and the droplet. The isothermal units $U U$ obtained from the measurement signal, represents the radiation flux received by the detector with reference to the blackbody one. On the measurement configuration, the image is formed by 200 lines and 200 points per line and the field of view is $6^{*} 6 \mathrm{~mm}^{2}$; the line frequency is $15 \mathrm{~Hz}$. The mode of operation, the image definition and the frequency, can be easily changed into the software.

\section{System characterization}

\subsection{Temperature calibration}

Referring to figure 2 , the detector first sees during the scanning of a unique line, an isothermal plate and then the blackbody. If $\phi(T)$ is the Planck relation between radiation flux and temperature, and $\varepsilon$ the emissivity, one can write the difference of radiation flux received from the blackbody (index $b$ ) and from the plate (index $p$ ). As the relation between radiation flux and isothermal unit is linear, that difference converted in measured isothermal units IUm gives:

$$
I U m=K \varepsilon_{b}\left\{\phi\left(T_{b}\right)-\left[\phi\left(T_{p}\right) \cdot\left(\varepsilon_{p} / \varepsilon_{b}\right)\right]\right\}
$$

where $K$ is a factor of proportionality. It follows that the ideal calibration law $I U$ for a ideal blackbody with emissivity equal to unity is:

$$
I U=\left(I U m / \varepsilon_{b}\right)+\left[K \phi\left(T_{p}\right) \cdot\left(1-\varepsilon_{b}\right)\left(\varepsilon_{p} / \varepsilon_{b}\right)\right]
$$

The shift introduced by the second term in (2) is negligible in our case. The calibration curve of the system is represented in figure 3.

\subsection{Temperature resolution : NETD}

In the same experimental conditions, if $V_{n}$ is the RMS voltage of the signal, we evaluates the NETD by the relation:

$$
N E T D=V_{n} /[\Delta V / \Delta]_{T_{b}}
$$

where $[\Delta V / \Delta T]_{T b}$ is the slope of the calibration curve at the temperature $T b$.

Figure 5 compares the NETD measured by the IR system and that one obtained from the model. The difference comes from the fact that we take only into account the photon noise in the simulation. In the experiment, electrical noise and parameter uncertainties have to be taken into account. However, the droplet temperature resolution for the experimental system, is lower than $0.1^{\circ} \mathrm{C}$ between $0^{\circ} \mathrm{C}$ and $60^{\circ} \mathrm{C}$ ( $\mathrm{Ta}$ : ambient temperature).

\subsection{Spatial resolution : $S R F$}

For the experimental conditions the slit response factor ( $S R F$ ) is about $500 \mu \mathrm{m}$ at $90 \%$ of attenuation. That value is due mainly to the size of the detector $(250 \mu \mathrm{m})$, to the diffraction effect which is important on that wavelength range and to the aberrations of the optical system.

\section{Temperature measurement of a fixed droplet}

In order to validate the IR measurement technique, we have compared this results with numerical and experimental ones. We worked on a methanol droplet whose diameter is about $1.2 \mathrm{~mm}$, fixed in still air at ambient temperature. The droplet is generated by a syringe and hanged under a microscope, on a thermocouple bead made from chromel and alumel wires of 100 microns in diameter. The heating of the droplet is performed by a $60 \mathrm{~mW}$ helium-neon laser and a mechanical shutter fixes the duration of the irradiation. After that period, the droplet cools and reaches an equilibrium temperature, which is below the ambient one due to the evaporation of the methanol. The temperature evolution of the droplet is measured simultaneously with the IR system and with the thermocouple. Furthermore, the temperature evolutions are compared to a 


\section{http://dx.doi.org/10.21611/qirt.1992.015}

numerical model. Experimentally, the thermocouple bead embedded in the droplet gives its temperature at the center. The agreement between the IR measurement and the thermocouple is very good as shown by figure 6 , the difference being lower than one degree centigrade. Theoretically, the model [2] computes the temperature variation of the droplet. Using correlations from the literature, we evaluate the heat transfer coefficient and the rate of evaporation. The energy balance at each time step, gives the equilibrium temperature of the droplet. The comparison between the IR measurements and the numerical results is shown in figure 7 , (reduced temperature $=\log [(T-T f) /(T i-T f)]$, where $T i$ and $T f$ are respectively the initial and final temperature). Once again, we can appreciate the very good agreement between both set of results, during the heating process by the laser and during the cooling phase, particularly for the time constant. The scattering of the experimental points at the end of the cycle is due to the noise in the IR system.

Finally the figure $A^{*}$ shows an image of the methanol droplet at the beginning and at the end of the heating and cooling cycle. The degree of space resolution is visible from this image and coherent with the measured one (cf 3.3).

\section{Conclusion}

A first simulation approach of an IR system devoted to droplet temperature measurement has shown the feasibility of that technique, with a temperature resolution lower than a degree.

The corresponding experimental system has been realized and characterized. It follows from the comparison with theory (models) and experience (thermocouple) that the ability of the system and its resolutions to measure the temperature of a vaporizing droplet, have been
demonstrated.

\section{Acknowledgements}

This work has been supported by the French Ministry of Defense (Direction des Etudes et Recherches Techniques). The authors are also grateful to N. Naudin and M. Plazanet for their appreciated help.

\section{REFERENCES}

[1] VAN DE HULST (H.C).-Light scattering by small particles.

Dover publications Inc., New York, 1981. [2] ABRAMSON (B.) and SIRIGNANO (W.A.).-Droplets vaporization models for spray
combustion calculations.

I.J.H.M.T., Vol 32, N9, 1989, p 1605-1618.

\footnotetext{
* The colour plates of this article 15 are located on page $I V$ of the colour gathering, at the end of the book
} 

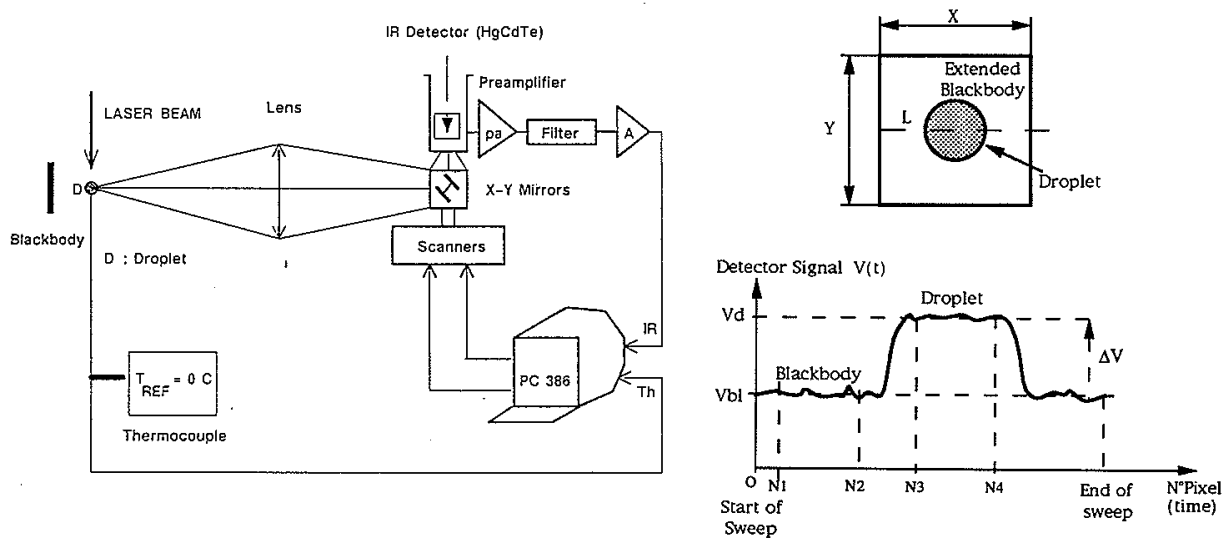

Fig. 1. - IR system measurement principle

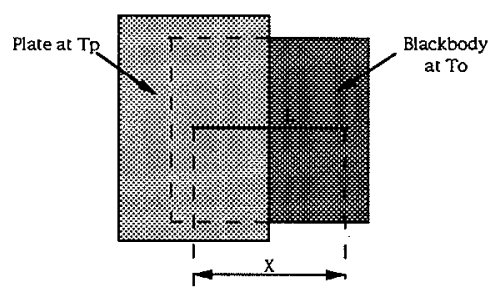

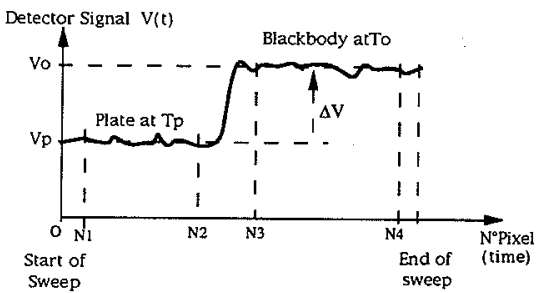

Fig. 2 - Calibration principle

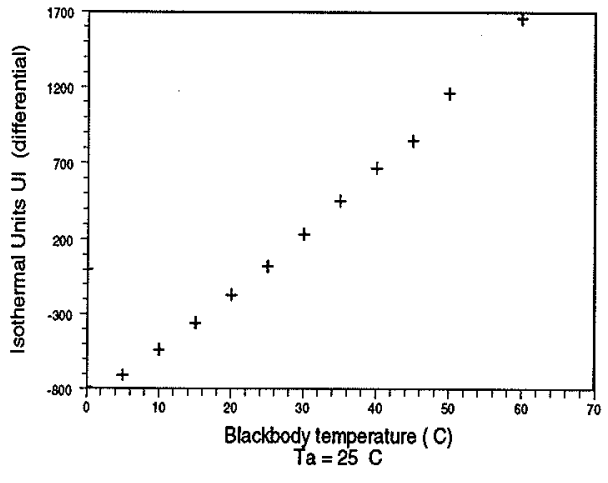

Fig. 3. - Calibration curve 
http://dx.doi.org/10.21611/qirt.1992.015

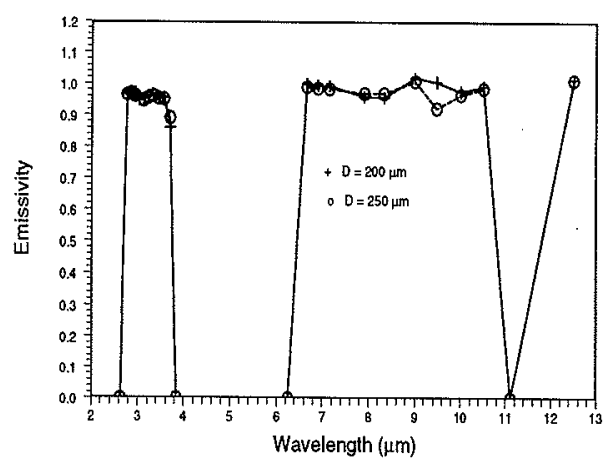

Fig. 4. - Spectral emissivity of methanol droplets

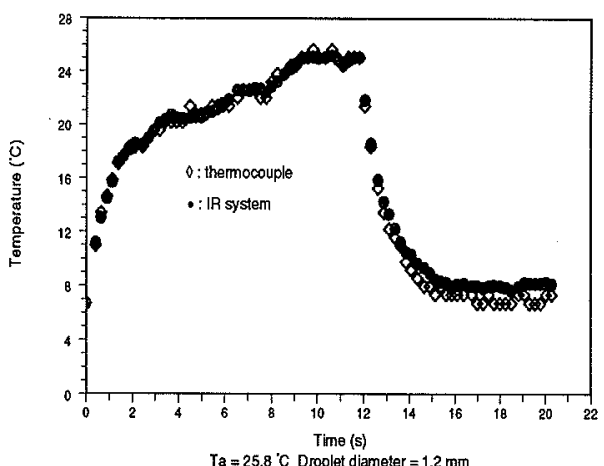

Fig. 6. - Methanol droplet cooling : IR and thermocouple measurements

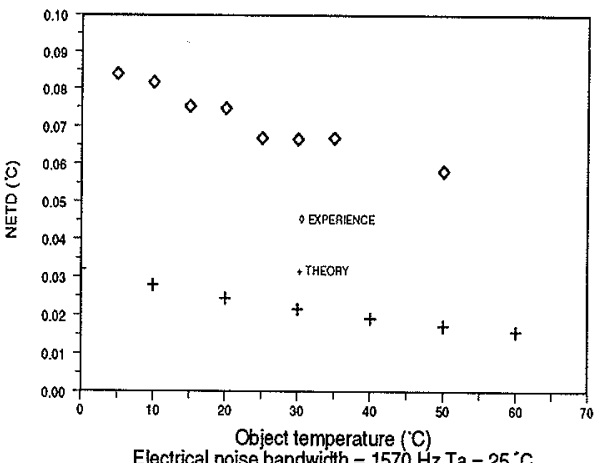

Electrical noise bandwidth $=1570 \mathrm{~Hz} \mathrm{Ta}=25^{\circ} \mathrm{C}$

Fig. 5. Theoretical and experimental NETD

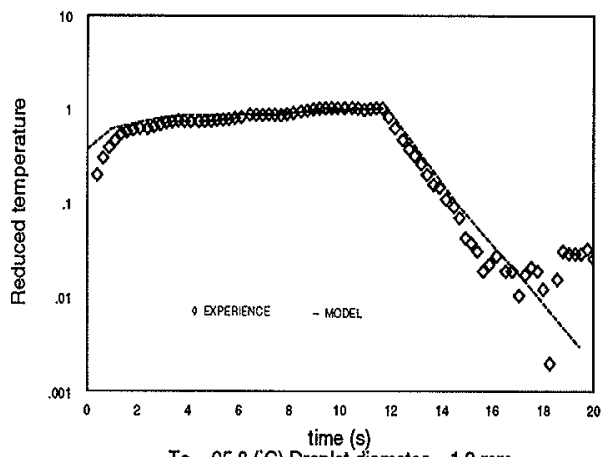

$\mathrm{Ta}=25.8$ (C) Droplet diameter $=1.2 \mathrm{~mm}$

Fig. 7. - Reduced temperature of the methanol droplets 